\title{
Moderating Redundant Traffic Generated by Topology Mismatch in P2P Networks
}

\author{
B. Lalitha \\ Department of CSE \\ JNTUCEA, Anantapur, India
}

\author{
Ch D V Subba Rao, Ph.D \\ Department of CSE \\ S V University, Tirupathi, India
}

\begin{abstract}
The formation of unstructured overlays without considering the underlying physical network properties introduces a substantial amount of redundant traffic into the P2P network. Also the frequent changes to the overlay topology and message overhead involved in maintaining the P2P overlays affects the performance of the system. By optimizing the routes traversed by the peers during the communication and implementing a simple but scalable and robust P2P overlay, the proposed system tries to reduce the redundant traffic in the network. The system is assessed by proper simulation and analysis with the findings providing improvements in the total system performance.
\end{abstract}

\section{General Terms}

Distributed Networks, Network Traffic, Simulation, Overlays

\section{Keywords}

P2P Overlay, Underlay, Simulation, Topology Mismatch Problem, Network Traffic

\section{INTRODUCTION}

In a peer-to-peer system each node acts as both as a client and a server as it gets services from other peers and also provide services to other participating peers. Initially peers constituting the $\mathrm{P} 2 \mathrm{P}$ overlay are independent from the underlying physical network i.e. internet. Peers communicate with other peers without considering their position or the relative distance from other peers in the network. This will result in a large communication delay between the peers and poor utilization of the underlay network properties and resources.

Formation of overlay network in unstructured networks like Gnutella 0.4 [1] involves generation of lot of messages by the flooding based routing and even in the systems which consider the location for overlay formation generates substantial amount messages to find the RTTs and delay between the peers.

The capability of peers constituting the P2P network varies by a large magnitude from one peer to the other. This inherent heterogeneity in the P2P system affects the total performance of the system as a small set of peers control a large part of the system resources.

So the major concerns which affect the performance of the P2P network are:
A. Network overhead due to Topology Mismatch problem: The topology mismatch problem is caused mainly due to the formation of the $\mathrm{P} 2 \mathrm{P}$ overlays without considering the properties underlying physical network. The properties include the location of the peer, the route traversed during the communication between peers, bandwidth etc.

Another reason for this problem is the random joining and leaving of peers in the network which affects the overlay topology and in turn increases the overhead for maintenance.

B. Traffic introduced during the Overlay Topology formation: Measurement studies [2] show that flooding based approaches generate about $330 \mathrm{~TB} /$ month in a Gnutella Network with only 5000 nodes and having $95 \%$ of the nodes at 7 hop distance.

Even in systems which consider the location of the node for the overlay formation additional messages overhead is involved to get the location information or delay of the peers.

C. Inherent heterogeneity in P2P system: All the deployed $\mathrm{P} 2 \mathrm{P}$ systems are characterized by heterogeneity in the participating peers. The distribution of peer properties such as processing power, storage space, and bandwidth and session duration are highly skewed. The greater part of peers has relatively less resources and small subset of peers control significant part of the total system resources.

In this paper the proposed system tries to solve the problem of reducing the network overhead without introducing too many messages for forming the overlay and improve the total system performance.

The rest of this paper is organized as follows. The work related to this paper is described in section 2 and the proposed system and overlay formation is presented in section 3 . Section 4 details the implementation and simulation of the system. Finally the summary and references are given in section 5 and 6 respectively.

\section{RELATED WORK}

Topology Mismatch Solutions: Numerous solutions [3] [4] [5] have been proposed to solve the topology mismatch problem considering the properties of the underlying physical network like the location of the peer or delay and RTT between the peers.

One of the earlier solution is LTM [3] tries to overcome the mismatch by sending a detector message in the proximity of the node. The peers which receiving the detector will record the relative delay from the sending peer. The solution provided is simple but as the size of the network increases the more detector messages will be flooded in the network which affects the scalability and performance of the system.

Another notable solution is Vivaldi [4] implements a distributed synthetic coordinate system and calculates the distance between two peers based on the coordinates assigned to the peer. But the overhead involved in implementing the coordinate system and calculating the relative distances which are not accurate compensates the merits provided by the solution.

To solve the mismatch problem SBO [5] uses the delay between the peers. But it is mainly used to reduce the 
optimization costs by distributing the optimization tasks to different peers. Peers in this system are characterized as red and white peers to distinguish the tasks performed by the peers. The demerits of this method are its complexity and message overhead involved for the overlay formation.

The solutions AOTO [6] and ACE [7] use the formation of multicast spanning tree approaches which forms an overlay multicast tree from the source peer to all its neighbor nodes. Each peer maintains a cost table containing cost to reach all its neighbors. Peers exchange the tables periodically to update the costs. The demerit of this approach is the overhead involved in maintaining and exchanging the cost tables and also the formation of the overlay multicast tree while maintaining the overlay topology.

Heterogeneity Solutions: The important method used to solve the problem of heterogeneity is the use of Super-peer topologies where a set of peers which are highly stable are provided additional responsibilities to improve the performance of the network.

The current stable version of Gnutella i.e. Gnutella 0.6 [8] uses the super peer topology where all the nodes are characterized as either ultra-peers or normal peers. The ultrapeer acts as server to the normal peers and the set of ultra peers form an independent sub network within the overlay network and handle most of the system functionalities.

The use of super peer topologies introduces many problems which are needed to be taken care of. The problems are listed below:

- System must decide the total number of super peers needs to be present in the system.

- Super peer election algorithm deciding which peer to act as a super peer.

- Dynamically adjusting the super peer set as the peers join and leave network.

- Clients have to be distributed and migrated dynamically.

- All the operations must be performed in a distributed manner in the dynamic environment.

Most of these problems will be addressed if the overlay is developed keeping in mind the dynamic nature of the P2P system. But the real demerit of the super topologies is that if a super peer fails all the clients connecting to that super peer gets isolated from the $\mathrm{P} 2 \mathrm{P}$ network.

Even though heterogeneity in P2P systems is a challenge it is also an opportunity which can be exploited to our advantage by giving more preference to the more stable nodes and improving the scalability of the system and allowing dynamic movement in the system. The proposed system uses this opportunity for forming the overlay network.

\section{PROPOSED SYSTEM}

The proposed system involves the implementation of a P2P overlay while reducing the overhead caused due to the three problems. The overhead introduced by the topology mismatch is optimized by using an underlay optimization solution called CJM. The problem of heterogeneity and message overhead is overcome by building a scalable and robust overlay at the overlay level of the P2P system.

\subsection{Common Junction Methodology}

Most of the solutions provided to solve the topology mismatch problem using the location of nodes, RTT's and delay involve optimizing the connections and neighbor selection at the overlay level. But the solution presented in [9] introduces the common junction methodology. The CJM method tries to reduce the network overhead at the underlay level by providing the improvements in the performance of the P2P system but provide more flexibility for the implementation of the overlay without considering the details of the underlying physical network.

The common junction methodology is the idea of finding the common junction between two paths through which the query is transmitted. The CJM can be depicted by the fig 1 .

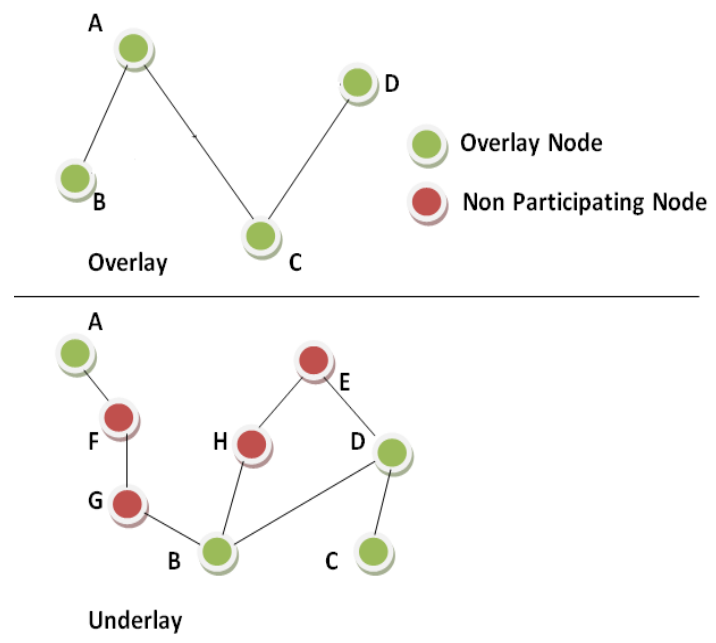

Fig 1: P2P Network

For a query to traverse in overlay through $\mathrm{B}->\mathrm{C}$ involves the path B $\rightarrow$ A $\rightarrow$ C. Since the actual query transmission occurs through the underlay network the same query transmission involves path $\mathrm{B} \rightarrow \mathrm{G} \rightarrow \mathrm{F} \rightarrow \mathrm{A} \rightarrow \mathrm{F} \rightarrow \mathrm{G} \rightarrow \mathrm{B} \rightarrow \mathrm{D} \rightarrow \mathrm{C}$

This path is considered as a combination of paths where the destination of first path is same as the source of the second path.

So the considered paths are $\mathrm{B} \rightarrow \mathrm{G} \rightarrow \mathrm{F} \rightarrow \mathrm{A}$ and $\mathrm{A} \rightarrow \mathrm{F} \rightarrow \mathrm{G}$ $\rightarrow \mathrm{B} \rightarrow \mathrm{D} \rightarrow \mathrm{C}$. The common junction is the ordered set of peers common in both the paths i.e. $C J=\{B, A\}$. In this manner the common junction is identified for any two available paths. Once the common junction set is found it is used optimized the route to traverse the query.

When the redundant path is identified in the form of common junction set, the query is routed through the non redundant path i.e. B $\rightarrow$ D $->$ C. In this manner the overhead caused by the topology mismatch problem is greatly reduced by the common junction methodology.

\subsection{Overlay Formation}

The overlay formation presented in this paper mainly deals to solve the heterogeneity inherent in the P2P system. The peer network suffers from poor performance if the problem of heterogeneity is not addressed. The structure of the overlay has to be adapted according to the capabilities of the participating peers. Otherwise the lowest performance peers with low processing power and throughput will become bottleneck to the system. 
The proposed system involves the use of a modified super peer topology and uses a value metric for the super peer election. The issues considered for the construction of this modified super peer network include

- Super peer election algorithm

- The number of super peers.

- The number of clients for each super peer.

- Issues related to the dynamic nature of the P2P network.

\subsubsection{Super Peer Election}

The super peer election algorithm constitutes the major function to find which peer in the network has eligibility to act as the super peer in the network. Gnutella 0.6 specifies that a peer can become a super peer if the peer has nonfirewalled link with $20 \mathrm{~KB} / \mathrm{s}$ download bandwidth and 10 $\mathrm{KB} / \mathrm{s}$ upload bandwidth running a suitable operating system.

The super peer election in the proposed system depends on the value metric. The value metric is a function calculated at the peer locally and gives the peer's ability to give resources to the $\mathrm{P} 2 \mathrm{P}$ system and provide services to other participating peers. This metric is a domain specific and imbibe the requirements of the application built above the overlay network. For example a file sharing application can set the value metric based on the function of peers upload bandwidth. All the peers with a certain super peer threshold will act as the super peers and the remaining peers will become the clients.

For example a value metric which can be considered for the super peer election is the client degree. According to the number of the clients a client threshold is set to make a peer as a super peer. Such a threshold is defined as a function in the following manner

$$
\mathrm{T}^{\mathrm{c}}(\mathrm{p})=\mathrm{N}-\mathrm{T}(\mathrm{p})
$$

Where $T(p)$ is the number of elected super peers, N-T (p) gives the degree of the clients and $\mathrm{T}^{\mathrm{c}}(\mathrm{p})$ gives the capacity of the peer to handle the clients. This capacity is set as the value metric such that

$$
\mathrm{V}(\mathrm{P})=\mathrm{T}^{\mathrm{c}}(\mathrm{p})
$$

Now each peer calculates the value metric locally and decides whether the peer can become the super peer.

\subsubsection{Number of Super Peers and Clients}

The number of super peers is very critical for the performance of the network. For example a file sharing system must have the number of super peers smaller enough to reduce the search traffic and large enough to handle the load of the clients.

In the proposed system the number of super peers is directly controlled by the super peer threshold. Larger the threshold smaller the number of super peers and vice versa. Since the number of super peers is considered by the threshold there will be no reconfiguration in the connections of the network.

\subsubsection{Dynamic Nature of the Network}

Due to the dynamic nature of the P2P networks if a super peer leaves the network all the clients which are attached to the super peer gets isolated from the network. This is a demerit in the Gnutella 0.6 specification.

In order support this dynamic behavior of the network the clients in the proposed system are allowed to connect to more than one super peer, thereby allowing the clients to be in the network even if one of the super peers leaves the network.
The algorithm 1 describes when a super peer will try to connect to other super peers.

$\mathrm{n} \leftarrow$ number of Super peer neighbors

if $0<\mathrm{n}<\mathrm{Smax}$

Return

If $\mathrm{n}>$ Smax then

$\mathrm{P} \leftarrow$ Select a random Super peer neighbor

Disconnect P

End if

If $n=0$ then

$\mathrm{Q} \leftarrow$ Search for other super peers

Connect Q

END if

\section{Algorithm 1: Super Peer Connections}

A super peer will be stable if it is connected to at least one but not more than Smax other super peers where Smax is defined as per the application requirement. In the same manner the algorithm 2 shows when a client changes its connection with the super peers.

$\mathrm{n} \leftarrow$ number of Super peer neighbors

if $n>0$

Return

If $\mathrm{n}>\mathrm{Nmax}$ then

$\mathrm{P} \leftarrow$ Select a random Super peer neighbor

Disconnect P

End if

If $n=0$ then

$\mathrm{Q} \leftarrow$ Search for other super peers

Connect Q

END if

\section{Algorithm 2: Client Connections}

A client will be stable if it is connected to connects to at least one super peer and not greater than $\mathrm{N}_{\max }$.

\subsubsection{Overlay Topology}

The modified super peer topology constitute the peers which have high value are logically present as the super peers of the topology while peers with lower value will be present as clients. In this topology value thresholds are defined to distinguish low value peers to the higher ones. Peers with similar thresholds are present close to each with small hop count in the overlay. According to the requirements of the application multiple thresholds will be set. The number of thresholds and the number of peers per threshold can be dynamically adjusted without changing the peer connections. A general overlay topology is depicted in the Fig 2. 


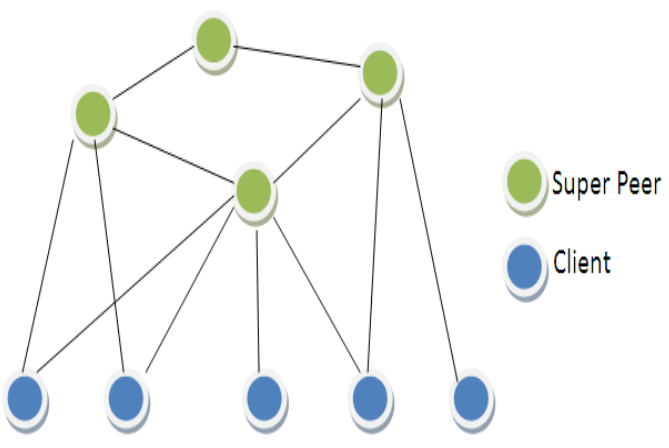

Fig 2: Overlay Topology

The overlay topology presented in the Fig 2 looks similar to one used to show the super peer topology but the client in this system has more than one connection to the super peers and the super peers are elected using the value metric.

The topology is independent from the application and requires that for each peer $\mathrm{P}$ in the system the value metric is assigned. This metric captures the application requirements. The higher value peers can be easily found by the lower ones using a search which routes the queries to the higher levels of the logical topology similar to a hill climbing approach.

\section{SIMULATION RESULTS}

Initially a general purpose java simulator is considered to implement the proposed system. In the system each node is assigned a value metric based on the requirement of the application. The proposed system considers a file sharing application, so the value metric considered is the accessible bandwidth of the peer. Then the initial number of super peers is set along with the threshold values which again hold the application requirements.

Each super peer in the network can have up to 32 clients and connections to other 30 other super peers. A threshold is set which states that a peer with more than $90 \%$ of connections can become a super peer. To evaluate the performance of the system results of the proposed system are compared with the stable versions of Gnutella i.e. Gnutella 0.4 and 0.6.

The metrics considered to evaluate include the hop count or average number of hops per query to evaluate the effect of the CJM methodology, query delay, traffic cost and success rate. The plot 1 describes the average number of hops taken by the queries during the communication of nodes.

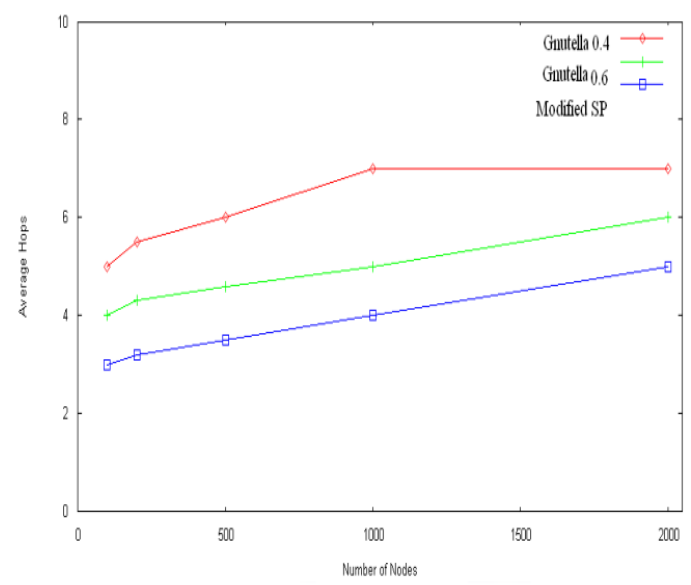

Plot 1: Average Hop Count
Plot 1 show that the major parts of the peers in the networks are not more than 7 hop distance. Since the CJM methodology optimizes the number of hops during the communication, so the modified super peer network has peers at not more than 5 hops away. The plot 2 describes the average traffic cost generated by the nodes. It shows that the average number of messages generated by the Gnutella networks gradually increases with the size of the network compared to the modified super peer network.

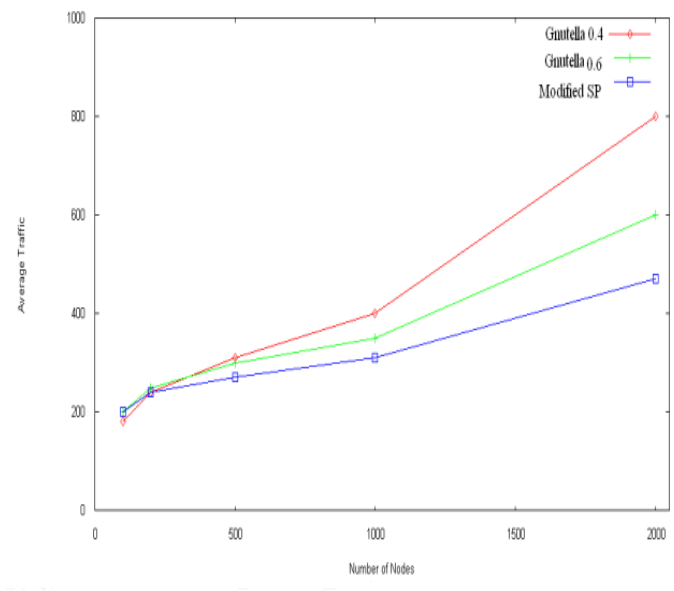

Plot 2: Average Traffic Cost

The plot 3depicts the query delay in the three networks. Since Gnutella 0.4 uses the flooding approach it takes more time to get the query results compared Gnutella 0.6. The CJM method optimizes the query traffic and replays the qeries faster than the other two.

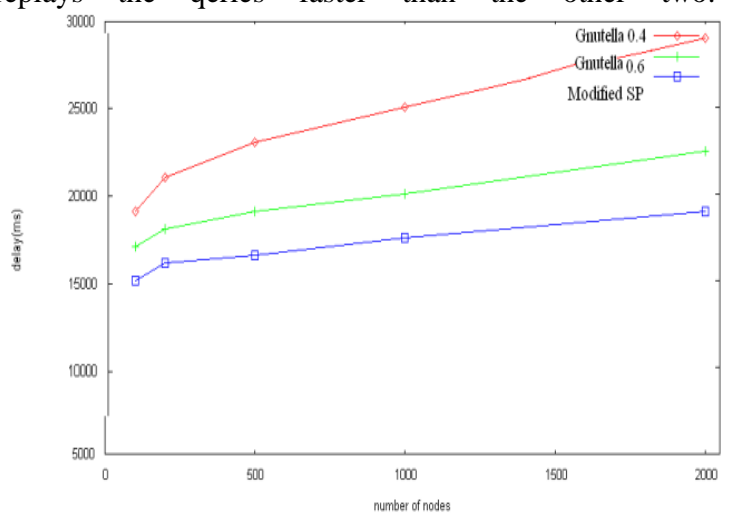

Plot 3: Query Delay

Table 1 depicts the query success rate of the three networks.

\begin{tabular}{|c|c|}
\hline Network & Success Rate \\
\hline Gnutella 0.4 & $98 \%$ \\
\hline Gnutella 0.6 & $86 \%$ \\
\hline Modified SP & $90 \%$ \\
\hline
\end{tabular}

Table 1: Success Rate

Since the Gnutella 0.4 uses the flooding based approach the query has high probability of finding the results compared to Gnutella 0.6 and the modified super peer system. 
The results show that the presented system reduces the network overhead caused by the topology mismatch problem and message overhead caused by the heterogeneity using the modified super peer network. The system provides faster query responses with low message overhead and reasonable success rate.

\section{CONCLUSION}

The proposed system implements a robust and scalable P2P overlay with improvements in the performance of the system and reducing the network overhead by using CJM with good query success rate. The future scope of this system would be to consider how the network traffic can be optimized with more improved overlays.

\section{REFERENCES}

[1] The Gnutella Protocol Specification 0.4, http://rfcgnutella.sourceforge.net/developer/stable/index.html.

[2] Ripeanu M. , Iamitchi A. and Foster I., "Mapping the Gnutella Network", IEEE Internet Computing, Vol. 6, No. 1, 2002, pp. 50-64.

[3] Li Xiao, Yunhao Liu, Xiaomei Liu, Lionel M. Ni \& Xiaodong Zhang.(2005).Location Awareness in Unstructured Peer-to-Peer Systems, IEEE Transactions on Parallel and Distributed Systems, No. 2, Vol. 16.
[4] Dabek, F., Cox, R., Kaashoek, F. \& Morris, R. (2004). Vivaldi: A Decentralized Network Coordinate System, Proc ACM SIGCOMM, 2004.

[5] Yunhao Liu, Li Xiao, and Lionel M. Ni, Building a Scalable Bipartite P2P Overlay Network, IEEE Transactions on Parallel and Distributed Systems, Vol.18, No. 9, September 2007, pp. 1296-1306.

[6] Y. Liu, Z. Zhuang, L. Xiao, and L.M. Ni, "AOTO: Adaptive Overlay Topology Optimization in Unstructured P2P Systems," Proceedings of IEEE GLOBECOM, 2003.

[7] L. Xiao, Y. Liu, and L.M. Ni, "Improving Unstructured Peer-to- Peer Systems by Adaptive Connection Establishment", IEEE Transactions on Computers, vol.54, pp.1091-1103, 2005.

[8] Tor Klingberg and Raphael Manfredi. Gnutella 0.6, 2002. http://rfc-gnutella.sourceforge.net/src/rfc-0_6draft.html.

[9] Bhushan S., Dave M., Patel R.B., "CJM: A Technique to Reduce Network Traffic in P2P systems", International Conference on Advances in Computer Engineering, 2010 . 